\title{
Flexible and extendable advance satellite tracking system
}

\author{
Mohamed Tarmizi Ahmad ${ }^{1 *}$, Michael Lam², Razali Abidin ${ }^{1}$, Shikin Razali ${ }^{3}$, Amzari Zhahir ${ }^{3 * *}$ \\ ${ }^{1}$ Department of Mechanical Engineering, Faculty of Engineering, Universiti Pertahanan National Malaysia, Malaysia \\ ${ }^{2}$ Worldgate Technologies, Menara UAC, No. 12, Jalan PJU 7/5, Mutiara Damansara, 47800 Petaling Jaya, Selangor, Malaysia \\ ${ }^{3}$ Department of Aerospace Engineering, Faculty of Engineering, Universiti Putra Malaysia, 43400 UPM Serdang, Malaysia \\ *Corresponding author E-mail:tarmizitomzee@gmail.com
}

\begin{abstract}
Aircraft tracking via satellite uses four dimensional aircraft position information to enhance flight safety by recording, scrutinizing and visualizing the information generated by an Airborne Tracking Unit (ATU) fitted onboard of aircraft flying from one point to another. It is an important device for aircraft operator to ensure its operational safety. Starfish Tracker SF3100-1 aircraft tracking unit can be used in aircraft satellite tracking applications. Research collaboration between Worldgate Technologies (WGT), Universiti Pertahanan Nasional Malaysia (UPNM) and Universiti Putra Malaysia (UPM) has developed the ATU originally from land base to airborne vehicles tracking, hence it provides multitudes of civil and military applications. The ATU is a unique device that enables multi-tasking application such as Air Combat Manoeuvring Instrumentation (ACMI), Network Centric Warfare and Flight Data Monitoring systems. In this paper, system characteristics and its applications are presented with the potential applications for Flight Data Monitoring system.
\end{abstract}

Keywords: satellite tracking; flight data monitoring; GNSS; GPRS; asset tracking; fleet management.

\section{Acronyms}

ACMI

FAA

FDM

GPRS

GNSS

ICS

IOT

ISCS

IMU

VNE

\author{
Air Combat Manoeuvring Instrumentation \\ Federal Aviation Authority \\ Flight Data Monitoring \\ General Packet Radio Service \\ Global Network Satellite System \\ Internet Communication Services \\ Internet of Things \\ Iridium Satellite Communication system \\ Inertial Measure Unit \\ Velocity Never Exceed
}

\section{Introduction}

FAA defines the Flight Operational Quality Assurance (FOQA) as a safety measure to enhance aviation safety by using recorded data during flight [1]. It provides guidance to aviation industries on the provision of Flight Data Monitoring (FDM) as a part of operator's accident prevention and flight safety program. Designated as the Starfish SF60ASGW Airborne Tracking Unit, this device can be employed in all sorts of flight data monitoring. The collaborative research between WGT and UPM/UPNM has extended the use of the mobile tracking unit from automotive to aviation applications and hence to multitudes of military and civil aviation applications of the GNSS/SATCOM asset management, ranging from private airplane to commercial and military aviation. Its telemetry infrastructure GPRS/Iridium/ICS provides low cost data transfer to the end user. This Advance Satellite Tracking System (ASTS) ensures secured connectivity at all time and optimization of operating cost. High-speed broadband spectrum enables its capability for multiple tasks.
This ASTS is unique in the sense that it facilitates multi-tasking capability such as Flight Data Monitoring and Tracking, and also Air Combat Manoeuvring Instrumentation (ACMI) and Network Centric Warfare. The Airborne Tracking Unit (ATU), as shown in Figure 1, has been developed with features that include the built-in magnetometer, gyros and inertial sensors, and GNSS, along with multiple digital, discrete and serial input/outputs. The operational test and evaluation (OT\&E) activities are still in progress as it is a flexible extendable system that can provide a platform for further research into other related disciplines.

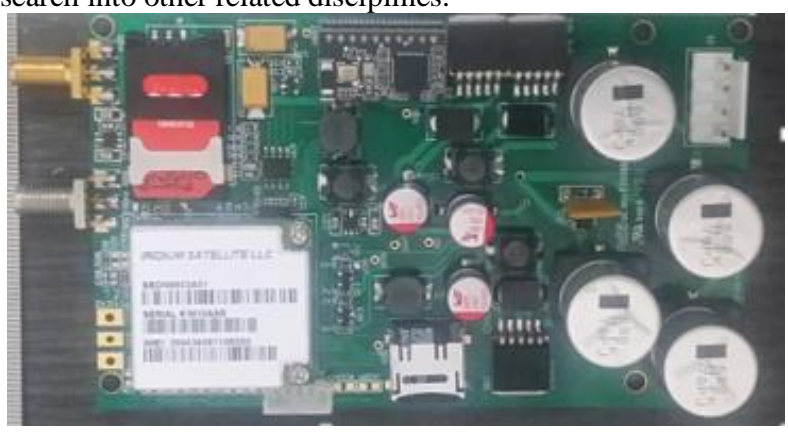

Fig. 1: Airborne Tracking Unit electronics

\section{System architecture}

The Starfish system architecture is illustrated in Figure 2, which is consisted of airborne segment (airborne tracking units, antennae and power supply), telemetry system (GPRS/GPRS/Wi-Fi /ISCS and ICS) and ground segment (end-user devices like computers, laptops and also smart phones). The system rides on the existing communication and IOT facilities, thus does not require any other infrastructures. 


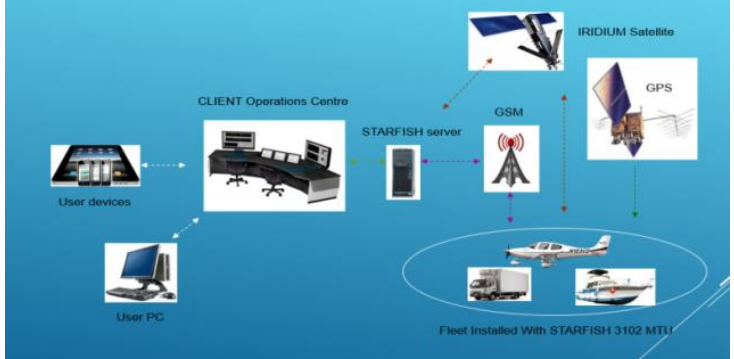

Fig. 2: System architecture (Courtesy of Worldgate Technologies)

\subsection{Device communications channel}

Data is sent from the device, via iridium and GPRS channels, to a Starfish server computer that resides in secured site. The messages are encrypted at the source before transmission and only decrypted at the Starfish server. The system auto switches between satellite and GPRS, with the priority given to GPRS. Location information is sent via satellite system when the device is out of GPRS range. High resolution and historical data is sent via the GPRS channel when the device is within range. Wi-Fi is used with devices within the cockpit such as cell phones, notepads and notebook computers with Wi-Fi connection. The Wi-Fi permits the maintenance and also updates of the tracking unit without having to remove it from the vehicle.

\subsection{Tracking strategy and features of SF60ASGW}

The unique features and tracking strategy of Starfish are described as follows:

- It practices a store and automatic forwarding strategy of high resolution data until GPRS is available to transport it back to the servers

- It implements a variable tracking period as opposed to a fixed tracking period by most trackers. This works in conjunction with its sensors that determine that a significant information exists and hence forces a tracking point and/or transmission. For example, a climb or descend and various other conditions

- With its own accelerometer, it is not required to be connected to the instrument panel in order to determine the orientation of the aircraft. This information is relayed back and the flight can be reconstructed based on ground speed, altitude, GNSS positioning, orientation, and acceleration

\subsection{Support equipment needed to implement the system}

It requires a server with good and reliable internet connection. At most, a mid-range server is required for the task. Other required devices includes computers, laptops or any smart phones or tablets with internet connectivity.

\subsection{Starfish fleet management systems}

Starfish can effectively facilitate the aircraft fleet management. Its tracking capability is developed with extra functions and it is also complemented with powerful software at command and control centre, which produces a high degree of control over the fleet. The Starfish ATU can also be described as a complex fusion of microelectromechanical systems (MEMS) gyros and accelerometers, magnetometers, C/A code and P/Y code GNSS receivers, and also advanced algorithm technologies and its telemetry via the GPRS/ satellite communications system [2]. Particularly, GPRS enables it to deliver data over the internet while stationary or in motion.

GPRS, being of higher capacity, faster data transfer and also cheap, makes Starfish cost effective for Fleet Management Applications. The Iridium satellite service is available for receivers worldwide. Nonetheless, the GPRS communication service is present where the connected network can cover and mostly, it is localized about $10 \%$ earth coverage. The Starfish covers this void with the Iridium
Short Burst Data Service (SB) that allows data delivery worldwide with about $110 \%$ earth coverage, which makes even ocean-going vessels and airborne vehicle could be tracked like any vehicle on land. Starfish's GPRS and Iridium SB are coexisting and they can automatically switched between them, enabling the operating cost to be minimized. The Starfish is an IoT device and fleet managers can access the flight data, maps, reports and analysis tools over the World-Wide-Web. The servers will provide cautions of any out of bounds conditions such as speed limits (stalls or VNE) or unusual attitudes such that action can be taken to prevent mishaps. These data allows fleet managers to execute analysis, forensic and fraud investigation just over the internet. If the suite of functions is not sufficient, data can be downloaded and special programs written to execute the task.

\section{Flight data monitoring system}

FDM is a systematic method of recording, retrieving, analyzing and acting upon information obtained from flight data to identify operational and/or maintenance risks before they lead to incidents and accidents. FDM incorporates various systems that continue to evolve to meet the unique requirements of each industry sector. All FDM systems are meant to improve the aviation safety [1-5] but the systems do differ when it comes to their specific areas of focus. In this respect, the Starfish ATU is a low cost substitute to the 'airplane communications addressing and reporting system' (ACARS) that is fitted to the commercial aircraft, and thus it is very beneficial for the general aviation operators. It has almost the same features as that of FDM.

\subsection{FDM system essentials}

The four essential aspects for a functional FDM program that are also available on the Starfish are listed as follows:

a) Capture and record flight data: The suitable FDM equipment must be procured and installed on each aircraft platform for the particular fleet. The equipment must be carefully chosen to ensure that the system's capabilities are matching the operator's actual requirements. There is a significant variation in capabilities, size, weight and cost between the various available FDM products. The ideal hardware should be lightweight, affordable, easy to install, easy to use, reliable, and capable of recording a wide variety of data. In reality, some compromises might have to be made when selecting the hardware for any particular application. The Starfish ASTS stores most of the data in the ATU flash memory and send flight significant data via satellite whilst the whole data package can be uploaded via the cheaper GPRS network.

b) Data extraction: The method used to extract data from the onaircraft hardware varies with different system manufacturer. Most will require the personnel to physically go to each of the aircraft to download the data. Starfish ATU automatically downloads their data (when the airplane is still airborne) via a cellular telephone network. Starfish ASTS sends flight significant data via satellite whilst the whole data package can be uploaded via cheaper GPRS network.

c) Data delivery: The data must be delivered, in a timely manner, to the facility that will analyse it. This data delivery can be such a logistical headache for operators that do physical downloads and operate from remote bases. The Starfish system provides low cost telemetry system to the end user. Excess to the WWW server can be done anytime, anywhere using internet based devices like iPad, iPhone, laptop and PC, as long as internet services are available.

d) Data analysis: Capability of the system software to properly analyze an operator's flight operations data is critical to the overall success of any FDM program. Most hardware manufacturers also supply the analysis software that is specifically developed for their products. Moreover, there are also independent software suppliers with products that can analyse downloaded data from a variety of hardware platforms. Another critical decision for any operator that 
is implementing FDM is whether to analyze their data in house or to outsource that function. Flight data of Starfish ASTS resides in operators' servers and the application software can be customized according to operators' requirements. For example, the speed and altitude data obtained from the Aeron M737 Flying ship as shown in Figure 3 and the tracks (i.e. flight path) are presented in Table 1 (sample data).

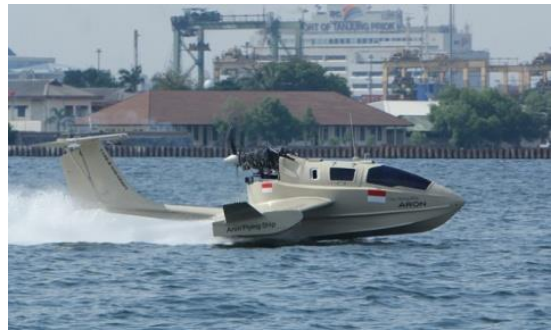

(a)

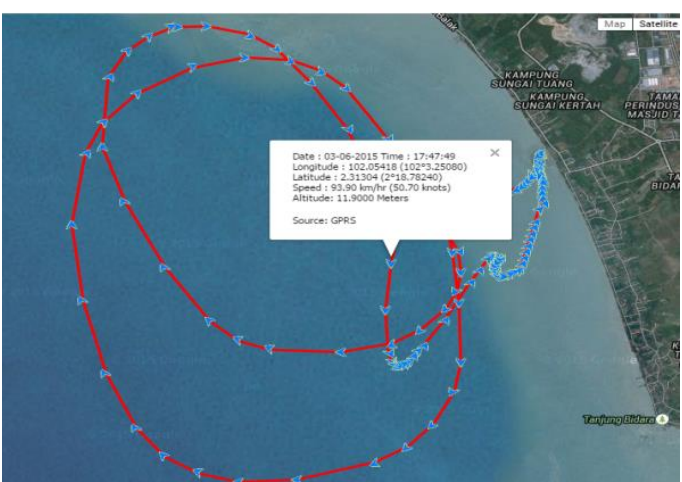

(b)

Fig. 3: (a) Aron M737 flying ship, and (b) its flight path

Table 1: Speed and altitude data

\begin{tabular}{|c|c|c|c|c|}
\hline Time & $\begin{array}{c}\text { Speed } \\
(\mathbf{k p h})\end{array}$ & $\begin{array}{c}\text { Altitude } \\
(\mathbf{m})\end{array}$ & $\begin{array}{c}\text { Speed } \\
(\mathbf{k t s})\end{array}$ & $\begin{array}{c}\text { Altitude } \\
(\mathbf{f t})\end{array}$ \\
\hline $17: 16: 23$ & 2.52 & 5.4 & 1.36 & 17.72 \\
\hline $17: 17: 36$ & 3.17 & 4.3 & 1.71 & 14.11 \\
\hline $17: 18: 17$ & 4.70 & 5.3 & 2.54 & 17.39 \\
\hline $17: 18: 24$ & 10.04 & 5.2 & 5.42 & 17.06 \\
\hline $17: 18: 44$ & 10.52 & 5.2 & 5.68 & 17.06 \\
\hline $17: 19: 04$ & 12.33 & 5.3 & 6.66 & 17.39 \\
\hline $17: 19: 12$ & 16.15 & 5.0 & 8.72 & 16.40 \\
\hline $17: 19: 26$ & 17.91 & 6.2 & 9.67 & 20.34 \\
\hline $17: 19: 45$ & 17.48 & 7.0 & 9.44 & 22.97 \\
\hline $17: 20: 05$ & 20.50 & 7.6 & 11.07 & 24.93 \\
\hline
\end{tabular}

The data are then plotted in Figure 4, which shows the vehicle in the takeoff (planning) and hopping phases. The other data such as accelerations, pitch, roll and yaw, magnetic compass data are also available, which can be displayed as Attitude Heading Reference System (AHRS) as depicted in Figure 5. The figure also shows the screen capture of the final approach of an airplane to the airfield runway 33 of Subang Airport. Even though there are many airfield features such as runway markings, taxiways, lights, buildings and roads, all of them are captured into the visual database explicitly.

The Starfish ATU is also installed and tested in EC135 helicopter as illustrated in Figure 6, which shows that it can track the flight vehicle in real time where the flight data are obtained via Iridium and GPRS telemetry system.

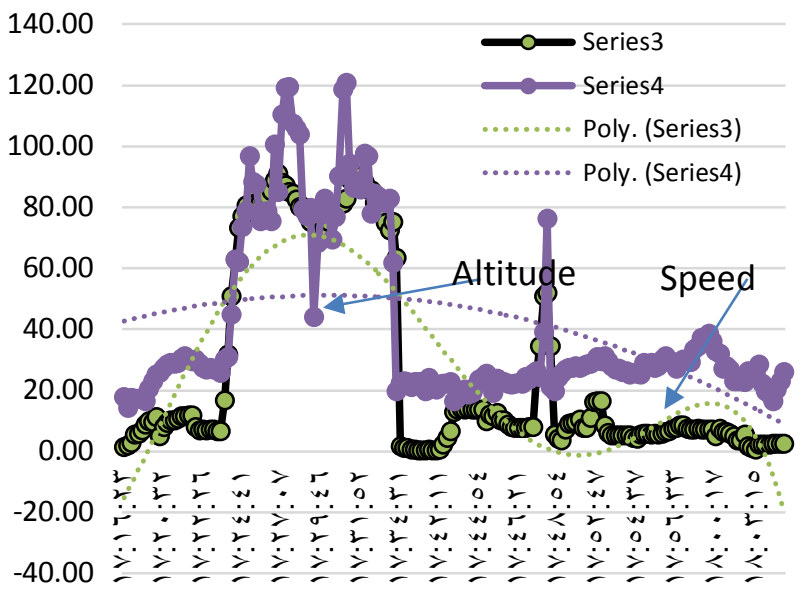

Fig. 4: Speed vs. altitude graph
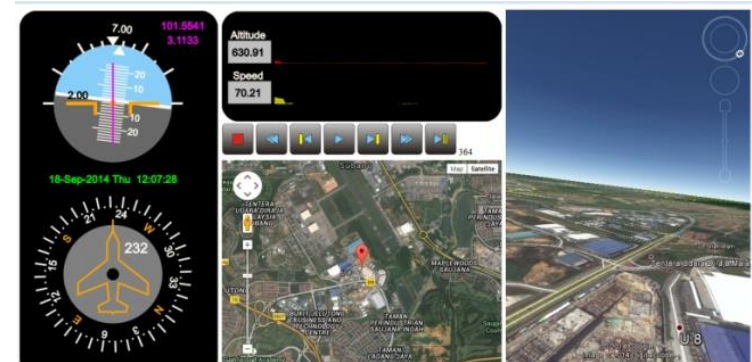

Fig. 5: 3-D Google Earth picture using data from onboard ATU

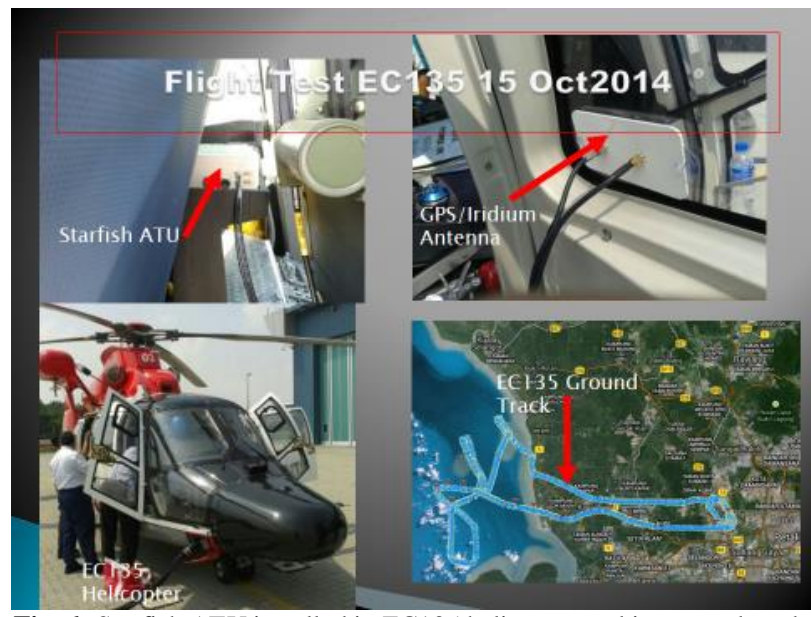

Fig. 6: Starfish ATU installed in EC135 helicopter and its ground track

\subsection{Overview of current FDM technology}

Today, new lightweight FDM hardware products are available in the market, along with multiple digital, serial and discrete input/ outputs; and they have several features like built-in GNSS receiver inertial, attitude, temperature sensor and also magnetometer [5]. If interfaced to a satellite communications transceiver, the details of any exceeding limit conditions can be notified to the management immediately after occurrence. Some FDM systems also provide audio and video inputs to record all flight deck activity, including instrument indications.

While FDM equipment is not designed to be crashworthy, it often does survive crashes, thereby providing the accident investigators with valuable data they would not have otherwise. The advantage of Starfish is that the data is kept in the server and can be retrieved, processed and replayed as required. Starfish ASTS is equipped with IMU, GNSS, magnetometer and also independent low power source (battery or solar powered), and it may be fitted with voice over internet communication in case of distress. Thus it does meet some of modern FDM system features. 


\subsection{Benefits of Starfish ATU}

Starfish provides a reliable and affordable solution for processing geo-positioning and telemetric data, collected and distributed by ATU installed onboard of airplane, overland vehicles and marine craft. Besides, it can also provide several benefits that enhance the aircraft location management and ensure the continuously updated operational status. This helps to improve both the efficiency of the field operations and also maintenance coordination. The system tracks mission ETA and collects mission time and distance usage, and then download the data into the connected business reporting/ invoice programs that supports concurrent web-users and secures the data transfer. It provides 'real time' flight tracking in 3D using Google Earth. It also provides the location of vehicle wreckage for Search and Rescue (SAR) mission in case of mishap [2].

\section{Discussion}

FDM provides a solution for many of the problems facing general aviation operators. Today, FDM brings increased level of safety and also sophistication to general aviation. In this paper, the basic framework of Starfish Flexible and Extendable Tracker has been introduced as a Flight Data Monitoring System that provides many benefits for aircraft operators including maintenance, operations, safety and training. A properly implemented and operated FDM system can control increasing maintenance and fuel costs while increasing safety margin. Operators using FDM programs should have competitive economic edge in very competitive marketplace.

\section{Conclusion}

FDM program helps operators to manage operational risks. In this paper, Starfish ATU that is a Flexible and Extendable Tracker has been introduced as a Flight Data Monitoring System, though it can provide additional facilities and services. The system performance is explained to identify the basic characteristics and highlight the system specification with potential of extension and improvement. The flight test done indicates that Starfish can offer a reliable and affordable solution for processing geo-positioning and IMU data, collected and distributed by the Starfish devices operating onboard of airplane, overland vehicles and marine craft. In addition, it can provide some benefits that enhance aircraft location management and ensures continuous updates of operational status. This helps to improve both the efficiency of field operation and the maintenance coordination. The system tracks mission end-to-end (ETE) data and collects mission time and distance usage, and then downloads the data into connected business reporting/invoice programs that support concurrent web-users and secure data transfer. It provides live flight tracking in 3D using Google Earth.

Databases with varying levels of fidelity have been developed and can be stored on a remote server. These databases carry the geospecific contents that are needed to assist the pilots in situational awareness, navigation guidance, and subsequently aviation safety. Navigation and communication data can be incorporated to drive the $3 \mathrm{D}$ visual display either autonomously or with awareness of any external traffic when it becomes available. The system will only operate under broadband system since connectivity with the internet is required to access Google Earth. However, the pace of innovation in IT development will push it to the next logical step i.e. move from developmental ensemble of hardware to embedded platform that can be commercialized. The software migration is straightforward while the hardware migration is only an exercise in integration.

\section{Acknowledgement}

This project is a private research arrangement between the main author and the WGT team (as shown in Figure 7). We would like to thank Universiti Putra Malaysia (UPM) for the financial support under Geran Putra Siswazah (GP-IPS/2016/9498300), National Defense University Malaysia and Worldgate Technologies (WGT) for their continuous support of this research project on a small scale while waiting for funding for development to full blown capabilities of Flight Data Monitoring system.

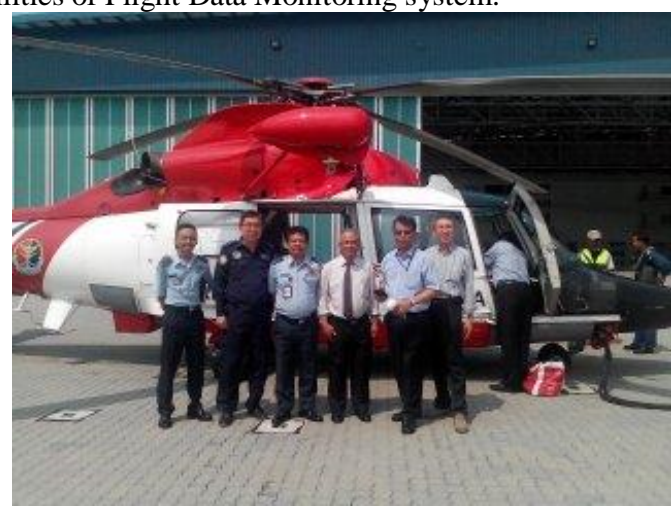

Fig. 7: Operational test and evaluation team

\section{References}

[1] Padmanaban S \& Mahendran SME (2015), Enhancing flight data monitoring and analysis can increase flight safety. Journal of Aeronautics and Aerospace Engineering 4(2), 1000143

[2] Ahmad MT, Lam M, Harithuddin ASM, Zhahir MAM \& Ariff OK (2009), Flight data monitoring/tracker system for search and rescue mission. Applied Mechanics and Materials 629, 350-354

[3] Civil Aviation Authority (2013), Flight Data Monitoring: CAP 739.

[4] International Civil Aviation Organization (2005). ICAO Accident Prevention Program.

[5] Burton A (2014), Aircraft tracking: What is currently available?, Apex Flight Operations. 